\title{
COMMISSION 31: TIME
}

\author{
(L'HEURE)
}

PRESIDENT: G. Petit

VICE-PRESIDENT: D.N. Matsakis

ORGANIZING COMMITTEE: D.C. Backer, G. Beutler, T. Fukushima,

S.M. Leschiutta, E. Proverbio, G.R. Qi, C. Veillet, G.M.R. Winkler \& Z.C. Zhai

\section{Abstract}

The relative frequency stability and the accuracy of atomic time scales, like International Atomic Time TAI, is now of order $1 \times 10^{-15}$ thanks to progresses in clock technology and in clock comparison techniques. Cold atom primary Cs standards have a stated accuracy of $1 \times 10^{-15}$ and a stability in the $10^{-16}$ region. Other cold atom clocks provide even better prospects, as well as clocks based on trapped ions. Frequencies based on optical and microwave transitions can now be compared with a similar or even better uncertainty thanks to femtosecond comb technology. Clock comparison techniques based on GPS (see http://maia.usno.navy.mil/gpst.html), or on dedicated Two Way technology provide adequate performance when averaging data over one or a few days, and should be improved to accompany the progresses of clocks.

Since 1999 , a number of organizations initiated a review on the future of the UTC system (insertion of leap seconds between TAI and UTC to keep $|U T 1-U T C|<0.9 \mathrm{~s}$ ). Several working groups have been initiated, notably by the International Telecommunications Union (Special Rapporteur Group (SRG) in the Working Party 7A), by the International Union of Radio Science, and by the IAU following Resolution B2(2000). No immediate conclusion may be foreseen but a consensus should be reached over the next triennium.

\section{Consultative Committee for Time and Frequency}

The CCTF held its 15th meeting on 20 and 21 June 2001. Following the discussions, five Recommendations were adopted and submitted to the Comité International des Poids et Mesures (CIPM). The list is the following:

Recommendation CCTF 1 (2001): Secondary representations of the second

A Working Group to propose a mise en pratique for the SI second has been established with the following terms of reference: To see that Recommendation CCTF 1 is implemented. To establish appropriate links with the Consultative Committee for Length. To present a list of proposed frequencies to CCTF at its next meeting. To consult on this matter with member laboratories of CCTF. This should provide an established mechanism for evaluating the performance of new types of frequency standards, and if a need to re-define the SI second arises, this body of evidence would contribute to that decision.

Recommendation CCTF 2 (2001): Time and frequency comparisons using GPS phase and code measurements (see section 4).

Recommendation CCTF 3 (2001): The meaning of the designation "k" in UTC(k) and $\mathrm{TA}(\mathbf{k})$

This Recommendation is to be passed to the International Telecommunications Union. Recommendation CCTF 4 (2001): Calibration of timing links for International Atomic Time 
Recommendation CCTF 5 (2001): Technical guidelines for manufacturers of GNSS receivers used for timing

Additionally discussed were the present form of UTC and mostly the interest of preserving the leap second (see section 5).

\section{Computation and Dissemination of TAI and UTC}

Reference time scales TAI and UTC have been computed regularly and published in the monthly Circular T. Definitive results have been available in the form of computer-readable files on the BIPM home page and on printed volumes of the Annual Report of the BIPM Time Section for 1999, 2000 and 2001, Volumes 12 to 14. Research concerning time-scale algorithms includes studies to improve the long-term stability of the free atomic time scale EAL and the accuracy of TAI. Studies are being undertaken to evaluate the feasibility of providing quasi real-time predictions of UTC and TAI. Some $80 \%$ of clocks are now either commercial caesium clocks of the HP5071A type or active, auto-tuned active hydrogen masers. Starting in January 2001, the value of the maximum relative weight of clocks in TAI has been fixed to $2 / \mathrm{N}$, where $\mathrm{N}$ is the total number of participating clocks (a value set to $2.5 / \mathrm{N}$ in July 2002). It was shown, using real clock data over three and a half years, that such a choice for the maximum relative weight leads to a better discrimination between the clocks and improves the stability of the resulting time scale. We can thus expect an improvement in the stability of EAL in the near future. Studies on the TAI algorithm continue. It has been proposed an estimator to quantify the reliability brought by the use of an upper limit of weights. It has been shown that it is possible to optimize this reliability estimator, thus defining a weighting scheme which is optimal in this respect. Tests using simulated and real data have shown that this optimal choice may be used in TAI computation. The medium-term stability of EAL, expressed in terms of an Allan deviation, is estimated to be $0.6 \times 10^{-15}$ for averaging times of twenty to forty days over the period January 1999 to June 2002.

To characterize the accuracy of TAI, estimates are made of the relative departure, and its uncertainty, of the duration of the TAI scale interval from the SI second as produced on the rotating geoid by primary frequency standards. Over 1999-2002, individual measurements of the TAI frequency have been provided by ten primary frequency standards including two Cs fountains (NIST-F1 and PTB CSF1). Thanks to PTB's effort to publish the detailed results of bilateral comparison with TAI, joint PTB/BIPM reports have been published. Starting 2001, such detailed reports are published in the Annual Report of the Time Section. Over 1999-2002, the global treatment of individual measurements has led to a relative departure of the duration of the TAI scale unit from the SI second on the geoid ranging from $0.2 \times 10^{-14}$ to $1.0 \times 10^{-14}$, with a standard uncertainty of order $0.2 \times 10^{-14}$. Because the current procedure for steering TAI does not seem to be sufficient to reduce this offset, studies are undertaken to set up new steering procedures that would provide a more accurate TAI without impeding its stability.

To provide accurate and precise time, the U. S. Naval Observatory (USNO) currently maintains an ensemble of 71 cesium-beam frequency standards and 15 cavity-tuned masers in three buildings in Washington, DC and at Schriever Air Force Base in Colorado. A nonoperational cesium-based atomic fountain has reached stabilities of $1.0 \times 10^{-15}$ at one day, and construction of a rubidium-based fountain has begun. Delivery of a mercury-based trapped-ion clock (LITE) from Jet Propulsion Laboratory (JPL) was accepted in July 2002. Improved timescale operations involving less aggressive clock characterization, better prediction algorithms, and gentler steering were developed in 2001. Since then UTC(USNO) stayed within $4.6 \mathrm{~ns}$ RMS of UTC; the RMS of UTC-UTC(USNO) over January-July 2002 was $2.6 \mathrm{~ns}$.

The USNO has improved its monitoring of GPS Time through the installation of calibrated TTR-12 receivers and the use of a raised antenna mount for multipath reduction. USNO has participated in Two Way Satellite Time Transfer (TWSTT) for the generation 
of TAI, and has calibrated its timing links with two European laboratories (NPL and PTB). It has participated as a contributor of clock information to the International GPS Service (IGS). USNO has also participated in a joint IGS/BIPM pilot project to develop carrier phase GPS time transfer, shown that continuous filtering can remove the day-boundary discontinuities evident in independent daily GPS carrier phase time-transfer solutions, shown that modifications to TurboRogue/Benchmark GPS receivers can make them time-stable in the presence of power outages and system resets, and developed a timescale for the IGS that is under evaluation to become an official product. USNO has one operational time-stable TurboRogue, and installed time-stable Ashtech Z12-3Ts at all three of its buildings.

\section{IGS/BIPM Time and Frequency Project}

The "IGS/BIPM Pilot Project to Study Accurate Time and Frequency Comparisons using GPS Phase and Code Measurements" was formed jointly by the International GPS Service (IGS) and the Bureau International des Poids et Mesures (BIPM) in early 1998 to investigate and develop operational strategies to exploit geodetic GPS methods for improved global availability of accurate time and frequency comparisons. Recent activites mostly fall into the following areas:

* Consultative Committee for Time and Frequency (CCTF) - At its 15th meeting, held 20-21 June 2001 at the BIPM (Sèvres, France), the CCTF adopted Recommendation 2 (2001) which supports the Pilot Project and encourages full participation by the timing labs contributing to UTC.

* Deployment of GPS receivers - The IGS network currently consists of about 300 permanent, continuously operating tracking stations globally distributed. Of these, external frequency standards are used at $\sim 40$ with $\mathrm{H}$-masers, $\sim 25$ with cesium clocks, and $\sim 15$ with rubidium clocks; the remainder use internal crystal oscillators. There are about 18 IGS stations currently (March 2002) colocated at timing labs, half of which participate in two-way satellite time transfer operations.

* Common-view files from RINEX data - Pascale Defraigne (ORB) has developed a procedure to use RINEX data from geodetic GPS receivers to form CGGTTS-format common-view observation files, the current standard for international time comparisons. This method aims to permit common-view time links using calibrated geodetic receivers to be introduced into BIPM's UTC computation.

* GPS data analysis - The IGS implemented a new method developed by Jan Kouba (NRCanada) and Tim Springer (AIUB) to combine satellite and receiver clock estimates from the IGS Analysis Centers (up to six). The clock values are sampled at 5-minute intervals and exchanged using the clock RINEX format, starting with GPS week 1086 (29 October 2000).

* New IGS time scale - A new internally realized time scale was developed by Ken Senior (USNO) to improve the stability of the IGS clock products, which are otherwise limited to about $2 \times 10^{-14}$ at 1 day and longer by the instability of GPS time. The consistency of the original IGS clock and orbit products is fully preserved in the new rereferenced clocks. Official adoption of the new time scale by the IGS is expected in the near future.

* Clock "densification" - It was agreed that Analysis Centers may augment their IGS submissions by using the precise point positioning method to determine clocks for receivers not used in their orbit solutions. In this way it is expected that all stations equipped with external frequency standards, especially all timing labs, can be included in the IGS clock products.

* Instrumental delays - Gérard Petit (BIPM) et al. have developed and demonstrated techniques to calibrate the instrumental biases of the Ashtech Z12-3T receiver, in both absolute and relative modes. In 2001 the BIPM began a campaign to circulate an absolutely calibrated Ashtech receiver to differentially measure the biases of similar receivers deployed at timing labs. 
* Future - It is expected that the Pilot Project will transition to permanent operational status within the IGS by the end of 2002. A longer period will be required to evaluate the usefulness of the IGS clock products in the work of the BIPM.

\section{The Future of UTC}

The ITU-R Special Rapporteur Group (SRG) was created within the ITU Working Party 7 a to study the question ITU-R 236/7 "The Future of the UTC Time Scale". At its May 2001 meeting, with regard to the use of time scales in satellite navigation systems, telecommunications systems, computer networking, broadcast services and scientific uses, the SRG categorised the options for the future of UTC and leap seconds as follows:

Option 1: Maintain the status quo:

Recommend use of UTC as currently defined. Clarify time scales available and considerations for use. More advanced notice and information availability. Creation of a navigation time scale.

Option 2: Modify leap second procedures or occurrence:

Increase tolerance of and enable longer prediction interval and lower frequency of leap second occurrence. Fixed interval adjustment with multiple leap seconds possible. Correction at predicted intervals based on deceleration model, re-evaluated at fixed intervals.

Option 3: Use of, or transition to, another time scale:

Existing TAI made more accessible. New navigation time scale may be needed for celestial users. New time scale based on re-definition of the SI second.

In order to gather information before developing its recommendations which are to be released at its meeting in October 2002, the SRG has released a general letter to both sectors of the ITU announcing the SRG and its objectives, and also published articles and notices in journals and newsletters.

To gather information, an URSI Commission J Working Group was formed, which conducted a poll (see http://rom.usno.navy.mil/archives/leapsecs.html). The Working Group was eventually extended to become an URSI group in 2002 .

At its 2001 meeting (see section 2), the CCTF conducted an informal poll among its members on the three options for the future of UTC presented by the chairman of the SRG group (see above). The opinions were almost equally shared between the three options.

The IERS Earth orientation Product Center conducted a survey among its users on the possible options concerning the future of UTC. 247 answers were received covering many fields from telecommunications to astronomy. While overwhelmingly satisfied with the current method of UTC determination (leap seconds) and the way it is announced (IERS Bulletin D), $24 \%$ were in favor of changing the system of leap seconds and $21 \%$ had no opinion. Among the possible solutions for a new system preference is given for a system without further leap second, with $31 \%$ choosing UTC with leap seconds fixed to the current number of 32 and $27 \%$ choosing the use of TAI.

\section{Workshops, Colloquia and Conferences}

Various meetings relating to the scope of Commission 31 were held. In addition to the usual meetings of the time and frequency community, the following may be mentioned (see http://www.bipm.org/IAU31/meetings.html):

The fourth International Symposium on time scale algorithms, sponsored by the USNO, the BIPM and the IEN, was held in Sèvres (France) on 18-19 March 2002. A special issue of Metrologia will publish the Proceedings.

G. Petit 